\title{
ESTUDO DO POTENCIAL ENERGÉTICO E CARACTERIZAÇÃO FÍSICO-QUÍMICA DOS RESÍDUOS DO PROCESSAMENTO DE UMBU CAJÁ (Spondia spp.)
}

\author{
T. S. E. PEREIRA ${ }^{1}$, B. F. M. L. GOMES ${ }^{2}$, P. T. TAVARES ${ }^{1}$, S. L. F. ANDERSEN ${ }^{1}$ \\ ${ }^{1}$ Universidade Federal da Paraíba, Departamento de Engenharia Energias Renováveis \\ ${ }^{2}$ Universidade Federal da Paraíba, Departamento de Engenharia Civil e Ambiental \\ E-mail para contato: silvia@cear.ufpb.br
}

\begin{abstract}
RESUMO - A umbu cajazeira pertence à família das Anacardiáceas, é considerada uma planta xerófila, híbrida natural entre o umbuzeiro (Spondias tuberosa Arruda) e a cajazeira (Spondias mombin L.). Pesquisas em torno do umbu cajá estão sendo desenvolvidas para desvendar as propriedades da espécie e alternativas para o aproveitamento agroindustrial, bem como energético. Neste trabalho a caracterização físico-química do resíduo sólido do processamento de umbu cajá foi realizada para avaliar o potencial deste resíduo como fonte alternativa e renovável de energia utilizando processos de conversão térmica. A caracterização físico-química se deu por meio de análise imediata, densidade aparente, microscopia eletrônica de varredura (MEV) e análise termogravimétrica (TGA/DTG). A análise do teor de cinzas resultou em 5,24\%; teor de material volátil de 69,02\%; carbono fixo de 25,59\%; densidade aparente de $1,46 \mathrm{~g} / \mathrm{mL}$ e poder calorífico superior de $18,63 \mathrm{MJ} . \mathrm{kg}^{-1}$. Por meio das curvas do TGA e DTG em atmosfera inerte observam-se três principais perdas de massa, havendo um consumo total de aproximadamente 78,5\%. Com poder calorífico superior a algumas biomassas comumente utilizadas para a geração energética, o umbu cajá pode ser considerado um potencial combustível a ser utilizado em processos de conversão térmica.
\end{abstract}

\section{INTRODUÇÃO}

A demanda crescente por energia tem gerado um grande desafio à humanidade: produzir energia com redução na geração de resíduos e emissões de carbono. É necessário o aperfeiçoamento de processos para que a geração de resíduos seja a menor possível. Dada tal necessidade, a biomassa tem ganhado destaque, pela sua importância energética e na mitigação de emissões de gases de efeito estufa.

Com economia predominantemente agrícola, o Brasil já ultrapassou a marca de 2,0 milhões de hectares dedicados ao agronegócio e é o terceiro maior produtor mundial de frutas (IBRAF, 2010). No nordeste a fruticultura é um setor de extrema importância devido à oferta de emprego e alimentos; além de ser uma região semiárida, onde poucas espécies conseguem se adaptar.

A umbu cajazeira pertence à família das Anacardiáceas e é considerada uma planta xerófila, híbrida natural entre o umbuzeiro (Spondias tuberosa Arruda) e a cajazeira (Spondias mombin L.) e por não ter espécie definida é denominada Spondia spp. (Giacometti, 1993). A origem da planta é desconhecida, entretanto, é considerada uma frutífera nativa do nordeste (Carvalho, 2010). 


\section{Congresso Brasileiro de Engenharia \\ Química em Iniciação Científica \\ Unicamp - Campinas - SP \\ 19 a 22 de julho de 2015}

Os produtos do umbu cajá podem ser utilizados para diversos fins alimentícios, diminuindo a quantidade de resíduos descartados. No entanto, com a expansão da fruticultura no nordeste brasileiro, da indústria alimentícia e da demanda por alimentos saudáveis e nutritivos, pesquisas em torno do umbu cajá estão sendo desenvolvidas para desvendar as propriedades da espécie e alternativas para o aproveitamento agroindustrial (Santos et al., 2010), bem como energético.

O objetivo deste trabalho foi caracterizar físico-quimicamente resíduo sólido do processamento de umbu cajá (bagaço) e avaliar o potencial deste resíduo para ser utilizado como fonte alternativa e renovável de energia, principalmente utilizando processos de conversão térmica.

\section{MATERIAL E MÉTODOS}

Para este estudo foi coletado bagaço de umbu cajá de uma indústria de sucos da cidade de João Pessoa - PB. Assim que coletado, o bagaço foi seco em estufa a $105{ }^{\circ} \mathrm{C}$, para posterior caracterização, portanto o teor de umidade não foi medido.

A primeira etapa para a caracterização físico-química foi a preparação das amostras. Os resíduos de umbu cajá foram secos, triturados e peneirados para que o diâmetro da partícula fosse menor do que $100 \mu \mathrm{m}$.

A caracterização físico-química se deu por meio de análise imediata, densidade aparente, microscopia eletrônica de varredura (MEV) e análise termogravimétrica (TGA/DTG). A partir da análise imediata determinou-se o teor de cinzas segundo a norma ASTM E1755 e o material volátil segundo a norma ASTM E872. O carbono fixo foi calculado por diferença como mostra a equação (1):

$$
C F=100-(\% \text { cinzas }+\% \text { voláteis })[\%]
$$

O poder calorífico superior do umbu cajá foi calculado a partir da equação empírica (2) (DEMIRBAS, 1997), que utiliza a análise imediata.

$$
P C=0,312(F C)+0,1534(V M)\left[M J . \mathrm{kg}^{-1}\right]
$$

A densidade aparente do umbu cajá foi feita utilizando o método da picnometria em água.

A análise de MEV foi realizada para o bagaço e as cinzas do umbu cajá e utilizando-se o microscópio eletrônico de varredura, Oxford Instruments, modelo LEO 1430.

A análise termogravimétrica (TGA) é uma técnica usada para avaliar a variação de massa da amostra em função do tempo ou temperatura (Denari, 2012). Já a termogravimétrica derivada (DTG) é a derivada primeira da TGA, onde a derivada da variação de massa em relação ao tempo $(\mathrm{dm} / \mathrm{dt})$ pode ser registrada ou em função da temperatura ou do tempo (Denari, 2012). Esta pode ser obtida a partir do mesmo ponto de massa residual da TGA. A análise termogravimétrica foi realizada utilizando o equipamento marca TA, modelo Q-50. As amostras de umbu cajá foram aquecidas a uma taxa de $10{ }^{\circ} \mathrm{C} \cdot \mathrm{min}^{-1}$ a partir da temperatura ambiente até $900^{\circ} \mathrm{C}$, em atmosfera inerte $\left(\mathrm{N}_{2}\right.$, pureza mínima $\left.99,996 \%\right)$ a um fluxo de $50,0 \mathrm{~mL} \cdot \mathrm{min}^{-1}$. A massa das amostras foi de aproximadamente $10 \mathrm{mg}$. 


\section{RESULTADOS E DISCUSSÃO}

Na Tabela 1 são apresentados os resultados das análises imediata, de densidade, e o poder calorífico calculado da amostra de bagaço de umbu cajá.

Tabela 1 - Análise imediata, densidade e poder calorífico superior do bagaço de umbu cajá.

\begin{tabular}{cc}
\hline Amostra & Umbu cajá \\
\hline Densidade aparente ${ }^{*}\left[\mathrm{~g} \cdot \mathrm{mL}^{-1}\right]$ & 1,47 \\
Cinzas $^{\mathrm{a},{ }^{*}[\%]}$ & 5,25 \\
Material Volátila, ${ }^{\mathrm{a}}[\%]$ & 69,03 \\
Carbono fixo $^{\mathrm{a},{ }^{*}[\%]}$ & 25,59 \\
PCS $^{*}\left[\mathrm{MJ}^{\mathrm{kg}}{ }^{-1}\right]$ & 18,64 \\
\hline
\end{tabular}

A análise do teor de cinzas resultou em 5,24\%. Comparando com o teor de cinzas de outras biomassas como a casca de café, 13,96\% (Vale, et al. 2007) e a casca de arroz, com o teor de cinzas de 15,51\% (Vieira, et al. 2011), percebe-se que o umbu cajá possui baixo teor de cinzas, o que é favorável para os processos de conversão térmica de energia. Segundo Ferreira (2013) as cinzas e a sua composição química, afetam o comportamento dos processos de conversão energética a elevadas temperaturas, pois, podem formar escórias e aglomerações, prejudicando os equipamentos dos processos.

O teor de material volátil de biomassa é geralmente elevado (acima de $60 \%$ ), e para o umbu cajá o teor de material volátil resultou em 69,02\%. Comparando o valor obtido neste estudo com valores encontrados na literatura como, por exemplo, com o da madeira de Cedrorana (Cedrelinga catenaeformis) em forma de maravalha - 83,19\% (Vale, et al. 2007) e com casca de arroz - 66,36\% (Paula, et al. 2011) verifica-se que este valor é comum, e pode indicar uma eficiência do processo de queima dos resíduos de umbu cajá.

O carbono fixo do resíduo de umbu cajá é de 25,59\%. Comparando este valor com o encontrado para Pinus pinaster e Eucalyptus globulus por Telmo et al. (2010), de 14,1\% e $13,3 \%$ e o encontrado no caule do milho - 20,47\% (Paula, et al. 2011), pode-se prever que se submetido à combustão, a queima do resíduo do umbu cajá é mais lenta que destas outras biomassas.

$\mathrm{O}$ resultado encontrado para a densidade aparente foi de $1,46 \mathrm{~g} \cdot \mathrm{mL}^{-1}$. Vale et al. (2007) apresentaram densidade de $0,14 \mathrm{~g} / \mathrm{mL}$ para a casca de grão de café e de $0,095 \mathrm{~g} \cdot \mathrm{mL}^{-1}$ para a madeira na forma de maravalha, todos analisados em base seca. Em termos energéticos, uma densidade elevada acarretará uma maior produção de energia na forma de calor por unidade volumétrica (Vale, et al. 2007). A densidade também pode determinar os custos de transporte e armazenamento da biomassa. Quanto mais densa a biomassa, menores serão os custos.

A partir do cálculo do PCS do bagaço de umbu cajá obteve-se o resultado de 18,63 MJ.kg-1. O valor encontrado é superior aos apresentados por Menezes (2013), para maravalha de Araucária (Araucaria angustifolia), PCS igual a 17,32 MJ.kg-1, e Pinus sem espécie definida (Pinus sp.), PCS igual a 17,23 MJ.kg-1, já Pérez et al. (2006) apresenta o PCS de duas espécies de eucalipto: 17,384 e 17,927 kJ.kg-1 para Eucalyptus globulus e Eucalyptus 
nitens, respectivamente.Isso mostra que o conteúdo energético do bagaço do umbu cajá tornao um potencial combustível a ser utilizado em processos de conversão térmica.

Por meio da análise de MEV observa-se na Figura 1 que a estrutura morfológica da amostra é formada por grãos de tamanhos e formatos diferentes. O bagaço (Figura 1.a), tem a forma de várias lamelas sobrepostas. Enquanto as cinzas do bagaço (Figura 1.b) apresenta uma morfologia esponjosa, ou mais porosa.

Figura 1 - Microscopia Eletrônica de Varredura a) bagaço de umbu cajá $(10 \mu \mathrm{m})$; b) cinzas de umbu cajá $(10 \mu \mathrm{m})$
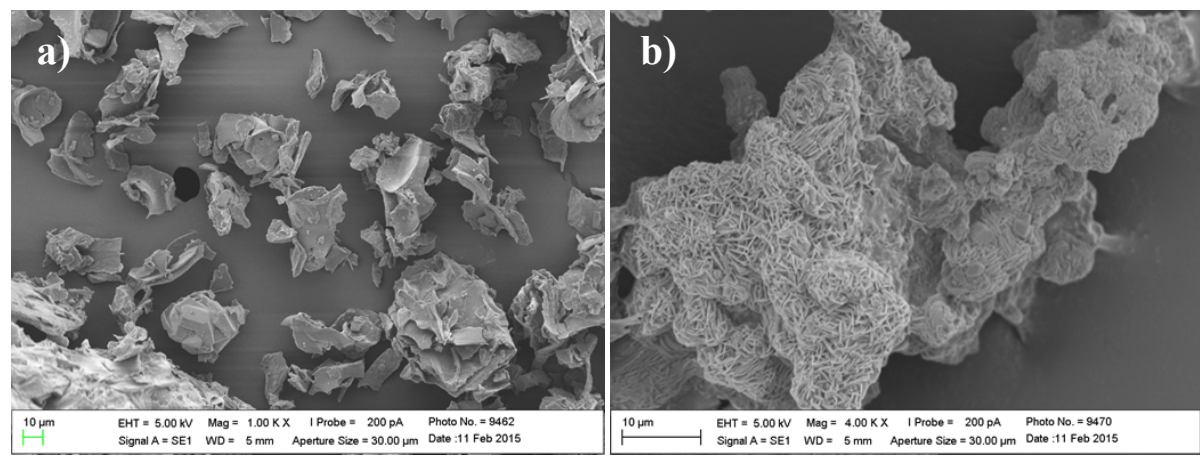

Quanto se trata de pirólise, a transferência de calor e as reações químicas influenciam a composição e os rendimentos do produto, as temperaturas de pirólise e as taxas de decomposição. Todos estes fatores variam com a composição da biomassa, devido à energia interna diferente de seus três constituintes poliméricos: hemicelulose, celulose e lignina. A celulose se decompõe rapidamente a produtos gasosos, a lignina se decompõe devagar e forma um conteúdo com elevado nível carbonoso e a hemicelulose se degrada em velocidade intermediária (Bach, 2006).

O comportamento do resíduo de umbu cajá submetido ao processo de pirólise é mostrado no termograma da Figura 2. A Figura 2 mostra a perda de massa em função da temperatura e mostra os picos de variação de massa com o tempo em função da temperatura (DTG).

Figura 2 - TGA e DTG do bagaço de umbu cajá submetido à pirólise

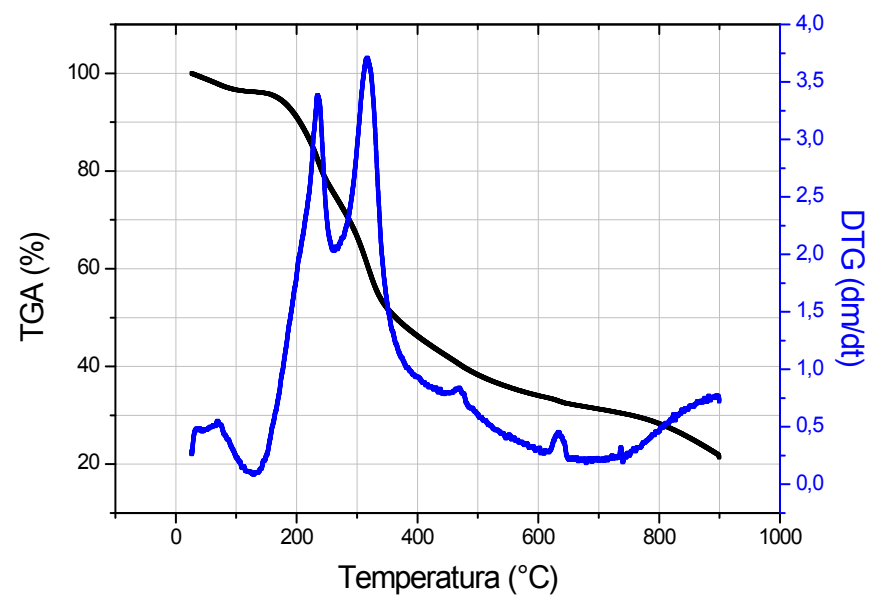




\section{Congresso Brasileiro de Engenharia \\ Química em Iniciação Científica \\ Unicamp - Campinas - SP \\ 19 a 22 de julho de 2015}

De acordo com a Figura 2, verificam-se três principais perdas de massa, a umidade residual, $4,3 \%$, foi perdida entre 32 e $155^{\circ} \mathrm{C}$ aproximadamente, já a perda de massa entre 155 e $345^{\circ} \mathrm{C}$ é referente ao início da degradação da matéria volátil de ligações químicas menos estáveis (em torno de 52,6\%), desta temperatura até $900{ }^{\circ} \mathrm{C}, 31 \%$ de material volátil mais estável foi degradado.

Observando a curva do DTG (Figura 2) verifica-se a ocorrência de três picos com maiores taxas de perdas de massa: o primeiro aos $235^{\circ} \mathrm{C}$, com uma variação de massa de $3,38 \% / \mathrm{min}$, o segundo aos $317^{\circ} \mathrm{C}$, com uma variação de massa de $3,70 \% / \mathrm{min}$ e o menor aos $469{ }^{\circ} \mathrm{C}$ com uma perda de $0,84 \% / \mathrm{min}$. Burhenne et al. (2013) citam para a madeira três fases de decomposição durante a pirólise: uma referente à hemicelulose, um pico durante a decomposição da celulose e um pico menor durante a volatilização da lignina, porém este mesmo estudo apresentou que a decomposição de três biomassas distintas (palha de trigo, bagaço de palha e madeira de abeto com casca) foi completa aos $557{ }^{\circ} \mathrm{C}$ para a biomassa herbácea e $627{ }^{\circ} \mathrm{C}$ para a biomassa lenhosa. Já Xin et al. (2013), analisando os três componentes principais da biomassa em forma de pó, extraídos da madeira de bétula, citam que o intervalo onde ocorreram as maiores taxas de perda de celulose foi entre 350 e $650{ }^{\circ} \mathrm{C}$; entre 50 e $75 \%$ da lignina foi transformada em biocarvão no intervalo de 750 e $850{ }^{\circ} \mathrm{C}$, confirmando a resistência deste componente à baixas temperaturas e possui alto teor de carbono fixo. Cortez et al. (2008) citam que com o aumento gradual da temperatura, como ocorre neste estudo, as reações favorecem a formação de frações sólidas em detrimento da gasosa e da líquida e, a medida que a temperatura de pirólise aumenta, os teores de hidrogênio e oxigênio diminuem e o teor de carbono aumenta na composição da fração sólida do combustível, consequentemente este tem seu poder calorífico superior aumentado.

\section{CONCLUSÃO}

Neste trabalho, o bagaço de umbu cajá foi caracterizado físico quimicamente para avaliar o potencial deste resíduo sólido como fonte alternativa e renovável de energia. O baixo teor de cinzas, de $5,25 \%$, é um resultado favorável para os processos de conversão energética e o carbono fixo é de $25,59 \%$, resultado similar ao encontrado na literatura, incluindo madeira de pinus e eucalipto. O poder calorífico superior foi calculado sendo $18,63 \mathrm{MJ} \mathrm{kg}^{-1}$, mostrando que o conteúdo energético do bagaço é adequado para que o mesmo seja utilizado em processos de conversão térmica. Os resultados das análises deste estudo validam a possibilidade de aproveitamento do potencial energético dos resíduos de umbu cajá.

\section{REFERÊNCIAS}

BACH, R. B. Ueber die strukture der oligomeren bestandteile von flash-pyrolyseaus biomass. Hamburg: Von-Melle-Park, 2006. Tese. Universidade de Hamburg, 2006.

BRASIL, Instituto Brasileiro de Frutas (IBRAF). Retrospectiva analítica 2010 - Cadeiaprodutiva das frutas. São Paulo, 2011.

BURHENNE, L.; MESSMER, J.; AICHER, T.; LABORIE, M. P.The effect of the biomass components lignin, cellulose and hemicellulose on TGA and fixed bed pyrolysis. Journal of Analytical and Applied Pyrolysis., v. 101, p. 177-184, 2013. 


\section{Congresso Brasileiro de Engenharia \\ Química em Iniciação Científica \\ Unicamp - Campinas - SP \\ 19 a 22 de julho de 2015}

CARVAlHO, L. D. Produção de geléia dietética de umbu cajá (Spondias SP.): Avaliação sensorial, fisica e fisicoquimica. 2010. 14 p. Dissertação (Mestrado Ciência de Alimentos) - Universidade Federal da Bahia, Salvador, 2010.

CORTEZ, L.A.B.; LORA, E.E.S.; GOMEZ, O. Biomassa para energia. Campinas, SP: Unicamp, 2008.

DEMIRBAS, A. Calculation of higher heating values of biomass fuels. Fuel, v. 76, n. 5, p. 431-434, 1997.

FERREIRA, A. C. O. Caracterização de vários tipos de biomassa para valorização energética. p. 3-5, 9, 10. 2013.

GIACOMETTI, D.C. Recursos genéticos de fruteiras nativas do Brasil. In: Simpósio Nacional de Recursos Genéticos de Fruteiras, 1992, Cruz das Almas. Anais... Cruz das Almas: EmbrapaCNPMF, 1993. p.13-27.

MENEZES, M. J. S. Poder calorífico e análise imediata da maravalha de Pinus (Pinus sp.) e Araucária (Araucaria angustifolia) de reflorestamento como resíduos de madeireira. Cascavel, PR: UNIOESTE, 2013.

PAULA, L. E. de R. e; TRUGILHO, P. F.; NAPOLI, A.; BIANCHI, M.L. Characterization of residues from plant biomass for use in energy generation. Cerne, v. 17, n. 2, p. 237, 2011.

PERÉZ, S.; RENEDO, C. J.; ORTIZ, A.; MANANA, M.; SILIÓ, D. Energy evaluation of the Eucalyptus globulus and the Eucalyptus nitens in thenorthof Spain (Cantabria). Thermochimica Acta, 451, p. 57-64, 2006.

SANTOS, M. B.; CARDOSO, R. L.; FONSECA, A. A. de O.; CONCEIÇÃO, M. do N. Caracterização e qualidade de frutos de umbu cajá (Spondias tuberosa x mombin) provenientes do recôncavo sul da Bahia.Rev. Bras. Frutic., Jaboticabal - SP, v. 32, n. 4, p. 1089-1097, Dezembro 2010.

TELMO, C.; LOUSADA, J.; MOREIRA, N. Proximate analysis, backwards stepwise regression between gross calorific value, ultimate and chemical analysis of wood. Bioresource technology, v. 101, n. 11, p. 3808-3815, 2010.

VALE, A. T. do; GENTIL, L. V.; GONÇALVEZ, J. C.; COSTA, A. F. da.Caracterização energética e rendimento da carbonização de resíduos de grãos. Cerne, Lavras, v. 13, n. 4, p. 416-420, 2007.

VALE, A. T. do; MENDES, R. M.; AMORIM, M. R. S. A.; DANTAS, V. F. de. S. Potencial energético da biomassa e carvão vegetal do epicarpo e da torta de pinhão manso (Jatrophacurcas). Cerne, v. 17, n. 2, p. 267-273, 2011.

VIEIRA, A. C.; SOUZA, S. N. M. de; BARICCATTI, R. A.; SIQUEIRA, J. A. C.; NOGUEIRA, C. E.

C.; Caracterização da casca de arroz para geração de energia. Revista Varia Scientia Agrárias, v. 3, n. 01, p. 51-57.

XIN, S.; YANG, H.; CHEN, Y.; WANG, X.; CHEN, H. Assessment of pyrolysis polygeneration of biomass based on major components: Product characterization and elucidation of degradation pathways. Fuel., v. 113, p. 266-273, 2013.

YANG, H.; YAN, R.; CHEN, H.; ZHENG, C.; LEE, D. H.; LIANG, D. T. In-depth investigation of biomass pyrolysis based on three major components: hemicellulose, cellulose and lignin. Energy and Fuels., v. 20, p. 388-393, 2006. 